\title{
BUILDING SHARED UNDERSTANDING DURING EARLY DESIGN
}

\author{
Danilo Gomes ${ }^{1}$ and Patricia Tzortzopoulos ${ }^{2}$
}

\begin{abstract}
Early Design Collaboration in construction projects can be hampered by misunderstandings between team members. Consequently, design actions are not supported by all, causing delays and frustration. This paper presents a study aiming to capture (a) misunderstandings between participants at early design stages, and (b) how these individuals resolved such misunderstandings through shared understanding. Anexploratory case study was conducted to investigate collaborative interactions of a Design Team, in an Architecture Office in San Francisco (USA). Data was collected during a concept design charrette focused at the building envelope of a Medical Office Building. Results from Protocol Analysis revealed misunderstandings emerging through independent actions and wrong assumptions among the participants, which triggered breakdowns in communication and the use of metaphors to construct shared understanding. This paper proposes a conceptual framework to explain the dynamics of shared understanding in early design stages, which could be used to help design teams to map, reflect about and improve their collaborative interactions.
\end{abstract}

\section{KEYWORDS}

Collaboration, Early Design, Shared Understanding, Social Construction, Design Team

\section{INTRODUCTION}

Early Design Stages, also known as 'Project Definition' according to the Lean Project Delivery System (Ballard 2008), consist of concept design generation supported by group decision-making. Thus, early design teamwork requires social interactions between design participants facing the challenge of aligning their activities to achieve better outcomes (Valkenburg 1998). In this context, collective design activities are a matter of expression of personal commitments and persuasion aiming for conflict resolution among participants (Cross and Cross 1995). Especially at early design, conflicts can emerge due to different language and forms of representation upon participants' different responsibilities and interests usually leading to lack of shared understanding on which design factor is most important in the task (Maher et al. 1996; Kleinsmann and Valkenburg 2008). Consequently, lack of shared understanding about the design object

PhD candidate in Architecture, University of Huddersfield, UK, danilo.gomes@hud.ac.uk

2 Professor, Associate Dean Research and Enterprise, School of Art, Design and Architecture, University of Huddersfield, UK, p.tzortzopoulos@hud.ac.uk 
and task can hamper the team's progress and can negatively influence the design outcome (Valkenburg 1998). The lack of shared understanding is linked to uncertainty and ambiguity within the design team, which is reflected in the lack of complete information to support collective design decisions (Hey et al. 2007). Designers use strategies to cope with the lack of input and progress with design activities (Koskela 2000), e.g. making decisions based on assumptions that should be checked later, but if these are incorrect, it leads to rework.

Hence, it can be said that one underlying characteristic of Early Design Collaboration is that designers usually have limited awareness and understanding of how other designers operate in the project and how their work has interdependencies with others in the design task (Cross and Cross 1995;Arias et al. 2000). Moreover, there is a poor understanding of what actions collaborators need to do when combining different design representations and how these actions can be implemented ( $\mathrm{Qu}$ and Hansen2008). Consequently, early design collaboration needs to be informed by a better understanding of how individuals create and negotiate representations and how this influence their shared understanding during the design activity (Snodgrass and Coyne 1992; Qu and Hansen 2008). Therefore, this paper addresses the following research questions: How misunderstandings emerge in early design? What participants do in practice to avoid and resolve misunderstandings?

\section{SHARED UNDERSTANDING}

In collaborative design, Valkenburg (1998) proposed that shared understanding is a mutual view amongst the team members on a relevant design topic and design activity. Thus, shared understanding involves similarities in the individual perceptions of actors about either how the design topic is conceptualised, as the content of the situation, or how their transactive memory system works, as the process to conceive a solution (Kleinsmann 2006). Transactive memory is a "set of individual memory systems, which combines the knowledge processed by particular actors with a shared awareness about who knows what" (Wegner1987 apud Valkenburg and Kleinsmann, 2008 p. 371). In this case, shared understanding faces the challenge of integrating various perspectives emerging from different descriptions of the world and, it depends on reasoning around conflicting arguments and goals among design participants (Arias et al. 2000).

Studying military coalitions, Smart (2011) proposed that shared understanding implies similarity of understanding in relation to a particular phenomenon (i.e. goals, task, situation), involving the emergence of the abilities to form expectations and predictions regarding future states, actions and events. According to Smart (2011), understanding is an ability to purposefully use knowledge in highly flexible, adaptive and contextsensitive ways. In this case, to think about knowledge in use can be considered problematic, especially considering the collective nature of knowledge (Snowden 2002). Traditionally, knowledge has been considering something that is dynamically created though movements between tacit and explicit states (Nonaka 1994). Consequently, knowledge management strategies have been conceived based on the idea that all knowledge could be disembodied from its possessors and become an organisational asset 
(Snowden 2002). For example, in the context of Lean Construction, Pasquire and Ebbs (2017), suggest that shared understanding, as the knowledge used in a project is something external to individuals and it should be managed as an organisational flow. However, recognisably, not all knowledge in the designers' head and conversations had, should or could be made explicit (Snowden 2002). A compelling argument on the epistemological difference between knowledge and understanding is found in Pritchard's (2014) work. According to the author, unlike knowledge, understanding is a specific kind of achievement, in which successes are accountable to ability. Hence, success should be creditable to the agent's exercise of the relevant ability (Pritchard 2014). More specifically, the author refers to a cognitive achievement, in which success (i.e. understanding) is creditable to the agent's cognitive ability. Thus, knowledge can be seen as less demanding than cognitive achievement, in situations in which to gain knowledge of causes does not require the agent to able to carry the relevant cognitive load by itself (i.e. knowledge is acquired by trusting the word of an expert) (Pritchard 2014). Understanding is more than a general conception on the relation of cause and effect, it requires a grip on how a cause generated an effect, a grip that could be given as an explanation of why the event happened (Pritchard 2014), and it can never be disembody from the actor and the situation. Hence, Smart's (2011) conception of shared understanding is key in design contexts to acknowledge the need for understanding in supporting the conception of expectations and predictions to change a problematic situation.

Therefore, adapting Bittner and Leimeister (2013) definition, shared understanding can be seen as the collective and dynamic ability to conceive and coordinate actions towards common goals or objectives ("meaning in use" or action perspective) of multiple agents within a group, based on diverse knowledge, beliefs and assumptions on the task, through the use of tools. Shared understanding is thus an enabler for collaboration, in which collective actions are complementary or compatible with each other, when evaluated against a common goal (Smart 2011). Furthermore, shared understanding is the expression of how agents' roles, responsibilities and capabilities (Smart 2011), are coconstructed in relation to the conception of shared goals and their perception of the aspects of the current situation. Therefore, shared understanding is a construct that is both a challenge and an important condition for team collaborative performance (Bittner and Leimeister 2013).

\section{BUILDING SHARED UNDERSTANDING}

Lack of shared understanding usually occurs because each participant interacts with their own set of assumption guiding their interpretations and actions (Hey et al. 2007). These assumptions seem to emerge from what Schön (1983) called frames, and Bucciarelli (1988) called object worlds, as underlying structures of belief, perception and appreciation, comprised of implicit understandings about what issues are relevant, what values and goals are important, and what criteria can be used to evaluate success. This are related to the concepts of common assumptions (Lloyd and Busby 2001) and common ground (Koskela 2015). 
Moreover, such frames will influence the implicit pairing between the individuals' perception of what is problematic in the situation and their conception of a desired end state of goal (Schön 1983; Hey et al. 2007). Divergent frames result in conflicting set of goals, assumptions and attentional foci in a design set (Hey et al. 2007).It could be said that, early conflict between individual frames may not prevent, but rather enable the negotiation of shared frames, as long as the conflict is made explicit (Hey et al. 2007) and the participants are keen to reflect on it. One problem is that individuals may not be aware of their own implicit assumptions until they are met with conflicting perspectives (Hey et al. 2007). According to Hey et al. (2007), several activities can make individual frames explicit and, consequently, reveal conflicts among them, like,for example, collectively building a group vocabulary and defining terms, concepts and categories. When conflicts between participants' individual frames are made visible, common frames can start to be negotiated (Hey et al. 2007). When individual frames are externalised, they expand the design team object, which allows other participants to expand their engagement in what Schön (1983) calls "conversation with the materials of the situation" (Arias et al. 2000).Here, metaphors can play a key role, because they embody symbolic representations that implies socially shared ways of perceiving a situation, as well as the ability to conceive changes in it (Tomelleri et al. 2015). According to these authors, metaphors are tools that build social relationships, generating consistency between an individual's inner world and their social environment, by unconsciously establishing a sense of performance, in terms of success and failure for the collective practice.

Therefore, following this constructivist approach to shared understanding and the type of interactions identified by Valkenburg (1998), we propose a framework to describe the dynamics building shared understanding in Early Design Collaboration (Figure 1). In the next sessions, we explain how the framework was used to identify designers' interactions towards shared understanding.

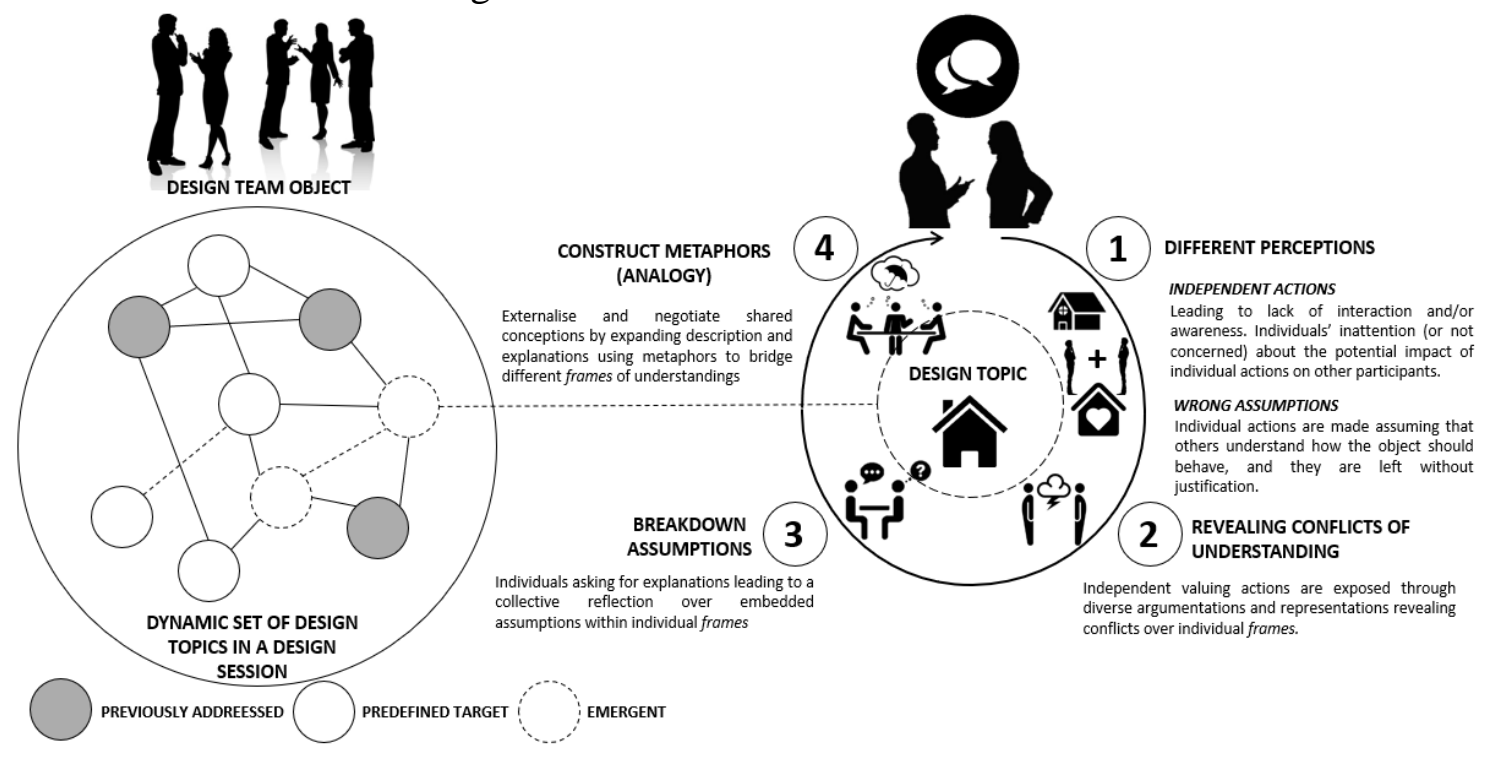

Figure 1: Dynamics of shared understanding in early design 


\section{RESEARCH METHOD}

This paper presents anexploratory study which is part of an ongoing $\mathrm{PhD}$ research on the dynamics of early design collaboration. A case study approach was adopted, in which an in depth empirical inquiry was conducted on a contemporary phenomenon in a real-world context (Yin 2014). By focusing on understanding the dynamics presented within a setting, a case study provides a description of the phenomenon that can support the test and generation of theory (Eisenhardt 1989). The aim of this study was to understand how misunderstandings emerge between design participants during early design and how these participants resolve such situations by building shared understanding. Thus, following an interpretive approach, this inquiry focused on the ways people make sense of the world through the inter subjectivity of shared meanings (Walsham 2006).

The exploratory study involveda design team working on a charrette at the schematic design of a Medical Office Building (MOB) in Alaska, USA. The participants, which were members of an Architectural Design Office, engaged in a one-day event, taking place at the company's office in San Francisco(USA). This was the third Design Charrette on this project. In order to anonymise the participants and the company studied, the real names of the participants were substituted by their professional role and a respective number.

According to the Project Design Leaders (Architect Leader 1, AL1, and Architect Leader 2, AL2) the first two Design Charrettes included the client, structural and systems engineers, the contractor, and they resulted in the initial scheme for the concept design of the MOB. The participants who engaged on this third Design Charrette are mostly from the same discipline. There were five architects: AL1, AL2, A3, A4, A5, and one Graphic Designer: GD, which came from a different office from the same company. According to the Brief presented, they were expected to contribute in exploring alternative ideas for the building envelope. During the Charrette, the participants interacted via verbal and graphical representations to explore different design ideas.

As an exploratory case study (Saunders and Thornhill 2009), the phenomenon was assessed to interpret what is happening according tothe proposed framework (Figure 1). Hence, observations in action and access to internal documents (i.e. brief, images, design representations), supported the understanding of people's shared meanings and issues. Protocol Analysis (Ericsson and Simon 1993) was used, in which the verbal and graphical interactions during the charrette were video-recorded and transcribed by the researcher for analysis against the constructs of the framework, in an attempt to make explicit the emergence and resolution of lack of shared understanding. The transcripts were coded according to the framework's constructs identifying interactions related to: different perceptions in the task (originated by independent actions and/or wrong assumptions);revealing conflicts of understanding; breaking down assumptions; and the use of metaphors (analogies). Thus, coding involved the examination of each segment of the protocol to determine whether it contained an explicit reference to aspects of shared understanding. 


\section{THE EXPLORATORY CASE STUDY}

The Design Charrette can be divided in three different modes of activity: briefing, exploration and presentation. In each of these modes, some of the interactions were identified describing actions leading to misunderstandings and the construction of shared understanding. The briefing activity started with the Project Design leader (AL 1) presenting the current state of design development based on the result from previous charrettes, in terms of conversations with the client and other stakeholders, as well as site analysis and initial design decisions. Following that, AL1 suggested that they should split into two groups to explore possible "design ideas" about the suggested design topics. Under this exploration mode, independent behaviour was noticed, in which the participants were exclusively involved in their own individual design activity (drawing and reflecting).At that time, there were some interactions to discuss emergent design topics, but less interactions in terms of contributing to shared representations (i.e. graphical artefacts).Finally, at the presentation mode, each of the participants presented their ideas to the group, using their sketches pinned in the wall.

It is possible to say that shared understanding was initially assumed and discussed in terms of the current state of the design task. AL1and AL2 seem to assume that everyone had similar understanding of what the design representations and images meant. Such assumption was also made about the meaning of the words used to focus and describe the selected Design Topics. As the Brief presented was conceived by AL1 and AL2, it is possible to argue that their frame, as their underlying structure of belief formed by AL1 and AL2 previous experiences, gave origin to certain assumptions on how and what the other participants should understand the current design situation and previous design actions. So, AL1 kick-started the activity presenting the Brief, as a document, showing and referencing the current "Foot Plan", as a design representation; hence commenting about the selected Design Topics, which were summarised in four keywords: Existing Campus, Punched Window, Curtain Wall + Glazing and Materials (Figure 2). Curiously, at that moment, the participants did not question these design objects (representation and concepts), which later revealed to be sources of misunderstandings. 


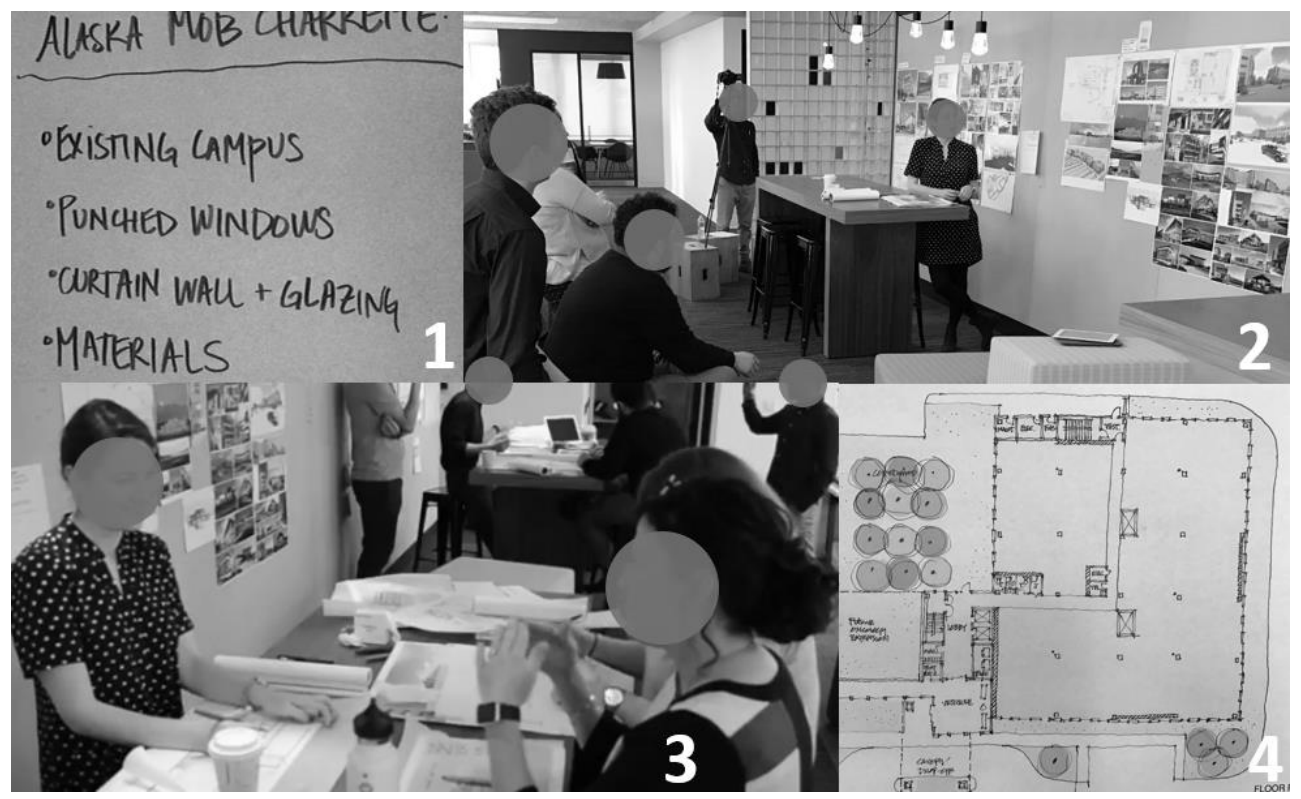

Figure 2: Elements of Design Situation (1,4); (2) Briefing and (3) Exploration modes

During the exploration mode, there was a moment when A4 indicated that she had a different interpretation about the representations (Foot Plan + Perspective) presented earlier, which revealed a conflict of understanding, as is indicated in Table (1). Then, the initial assumption that everybody was interpreting the representations similarly was proved wrong. In this case, the misunderstanding may have been caused by A4 lack of understanding of the representation or her inattention to some aspects of the representation. However, after this revelation, A4 and AL1 continued working on their individual conceptions of an Emergent Design Topic: the "Building Entrance". Later in the task, during the presentation mode, this lack of shared understanding was exposed again but in depth to the whole group, which supported the breakdown of the assumptions. At this stage, the participants engaged in a set of questioning and explanatory actions to collectively understand the reasons behind these diverse interpretations, and what were the assumptions embedded on these perspectives (Table 1).

In another moment, during the exploration mode of activity, AL1, A4 and GD started a playful conversation about the idea of a "pyramid" (which was suggested by the client as a reference to pyramids in the existing buildings in the campus) and how in their view it would not fit to the current concept of the proposed building envelope design. Through this open chat, GD suggested the idea of putting a pyramid as a sculpture in the courtyard.

However, till that moment, the idea of a "pyramid" was still a loose concept in the project and the discussion of their individual views of the purpose of the "pyramid" had not been explored. Thus, GD tried to build shared understanding about this Emergent Design Topic: the "Pyramid", by questioning and then discussing her conception of a Sculptural Element with AL1. The conversation was developed and led to the collective evaluation of her conception of how a "Sculpture" as the proposed objectification of the Emergent Design Topic: "Pyramid" could be implemented (Table 1).Interestingly, in this 
case, in addition to the use of graphical representations she also uses gestures to describe her conception (Figure 2, Image 3).

The use of a metaphor to bridge and convey understanding on design topics was noticed a few times during the Charette. The most significative happened at the end of the briefing, when AL1 try to explain her perspective about how they should approach the main objective of the session: design the exterior of the MOB. In her concluding argument she uses two metaphors within a comparison (the "cousin" vs. the "twin" building) to make it clear that the new MOB should fit into the context of campus, but at the same time it should not look exactly the same as the existing buildings (Table 1).

Overall, when participants didn't act towards exploring the diverse and conflicting understandings that emerged during the task, shared understanding could not be constructed. For example, during the presentation mode, while participants had the chance to present and provide explanations about their individual perceptions and conceptions over the design topics, they lacked the initiative to collectively reflect over how these ideas could be evaluated, balanced and, eventually, merged into a group proposal.

Therefore, building shared understanding requires that participants perceive and embrace the dialectical nature of collaborative design, and work upon contradictions emerging from different perceptions founded on individual frames that manifests through diverse artefacts of interaction (i.e. verbal argument, graphical representations, organisational processes). Through such dialectical activity participants in the early design collaboration can potentially review, reposition and construct, in the sense of a bridge, their collective interpretations and actions. 


\section{Table 1: Capturing the dynamics of building Shared Understanding}

Capturing the Dynamics of construction of shared understanding in Early Design Stage

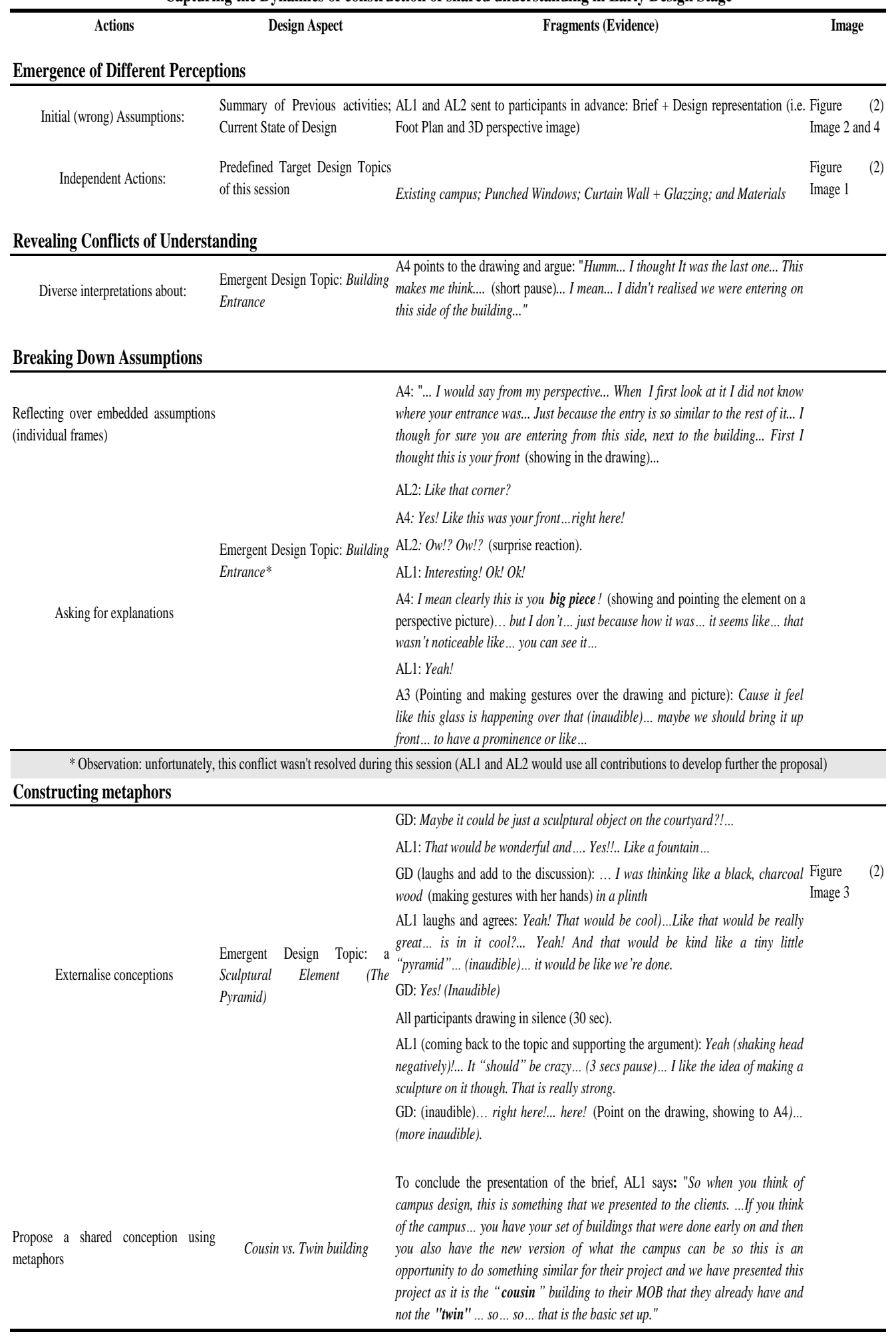




\section{CONCLUSIONS}

In conclusion, misunderstandings can be considered a natural emergent feature of early design collaboration, which may lead participants to engage in a dialectical construction of the activity. In this case, the emergence of diverse understandings in a design task is not a bad thing. However, if not revealed and resolved it can lead to wrong assumptions and expectations among the design team. Diverse understandings emerge and are manifested within the artefacts the participants design and choose to use in the task. When these diverse understandings are revealed and exposed in time, it can lead to an opportunity to explore and expand different ways to perceive the situation, as well as conceive different design alternatives.

However, exposing different perceptions in a design task requires participants to engage in collective explanatory interactions (e.g. asking and describing) involving reflective practices (i.e. why am I thinking in this way?), which help them breakdown assumptions, that may be proven to be wrong, especially about other participants' work contributions. Consequently, this means that initiatives that only focus on "exchanging information", as way to share knowledge about different perceptions and alternative conceptions is not enough. According to the proposed framework, design participants need to engage in explanatory actions (constructing "explanatory artefacts") that help them "bridge" different frames of understanding (i.e. object worlds), and collectively 'establish predictions about future states, in order to collaborate. Designers do that by using metaphors.

Therefore, this framework could be used to help design teams map and reflect about their interactions at early design. By focusing on setting the design team object (i.e. what are the specific design topics on this task?), individuals can expose diverse interpretations over design objects, and establish the means (artefacts) to evaluate them collectively.

Following this study, the next step on the research is to explore how the collective construction of the design object interacts with the collective construction of the project activity. This will be done to identify if different collaboration conceptions could also be a source of misunderstandings.

\section{REFERENCES}

Arias, E., Eden, H., Fischer, G., Gorman, A., and Scharff, E. (2000). Transcending the individual human mind-creating shared understanding through collaborative design. ACM Transactions on Computer-Human Interaction (TOCHI), 7(1), 84-113.

Ballard, G. (2008). The lean project delivery system: An update. Lean Construction Journal, 2008, 1-19.

Bittner, E. A. C., and Leimeister, J. M. (2013). "Why Shared Understanding MattersEngineering a Collaboration Process for Shared Understanding to Improve Collaboration Effectiveness in Heterogeneous Teams." System Sciences (HICSS), 2013 46th Hawaii International Conference. 106-114.

Bucciarelli, L. L. (2002). Between thought and object in engineering design. Design studies, 23(3), 219-231. 
Cross, N., and Cross, A. C. (1995). "Observations of teamwork and social processes in design.” In: Design studies, 16(2), 143-170.

Eisenhardt, K. M. (1989). Building theories from case study research. Academy of management review, 14(4), 532-550.

Ericsson, K. A., and Simon, H. A. (1993). Protocol analysis. Cambridge, MA: MIT press.

Hey, J. H., Joyce, C. K., and Beckman, S. L. (2007). Framing innovation: negotiating shared frames during early design phases. Journal of Design Research, 6(1-2), 79-99.

Kleinsmann, M. (2006). Understanding collaborative design, PhD. Thesis, Delft University of Technology, Delft.

Kleinsmann, M., and Valkenburg, R. (2008). Barriers and enablers for creating shared understanding in co-design projects. Design studies, 29(4), 369-386.

Koskela, L. (2000). An exploration towards a production theory and its application to construction. VTT Technical Research Centre of Finland.

Maher, M. L., Cicognani, A., and Simoff, S. (1996). "An experimental study of computer mediated collaborative design." In: Enabling Technologies: Infrastructure for Collaborative Enterprises. Proceedings of the 5th Workshop. 268-273.

Nonaka, I. (1994). A dynamic theory of organizational knowledge creation. Organization science, 5(1), 14-37.

Pasquire, C. and Ebbs, P. (2017). Shared Understanding: The Machine Code of the Social in a Socio-Technical System.In: 25th Annual Conference of the International Group for Lean Construction. Heraklion, Greece,pp 365-372.

Pritchard, D. (2014). Knowledge and understanding. In Virtue Epistemology Naturalized (pp. 315-327). Springer International Publishing.

Qu, Y., and Hansen, D. L. (2008). "Building shared understanding in collaborative sensemaking". In: Proceedings of CHI 2008 Sensemaking Workshop, Florence, Italy.

Saunders, M. L. P. and Thornhill, A. (2009). Research methods for business students, 5.

Schön, D. A. (1983). The reflective practitioner: How professionals think in action. London: Temple Smith.

Snodgrass, A., and Coyne, R. (1992). Models, metaphors and the hermeneutics of designing. Design Issues, 9(1), 56-74.

Snowden, D. (2002). Complex acts of knowing: paradox and descriptive selfawareness. Journal of knowledge management, 6(2), 100-111.

Smart, P. R. (2011). Understanding and shared understanding in military coalitions. Available at https://eprints.soton.ac.uk/267735/ (13/02/2018).

Tomelleri, S., Lusardi, R., \&Artioli, G. (2015). The metaphors of collaboration, or the social construction of collaborative interactions between health professionals. Acta Bio MedicaAteneiParmensis, 86(1Suppl), 7-18.

Valkenburg, R. C. (1998). "Shared understanding as a condition for team design." In: Automation in construction, 7(2), 111-121.

Walsham, G. (2006). Doing interpretive research. European journal of information systems, 15(3), 320-330.

Yin, R. K. (2014). Case study research: Design and methods. Sage publications. 